\title{
The role of antibiotic misuse when treating pneumonia in Somali regional state in Ethiopia: safety and efficacy awareness
}

\begin{abstract}
Whilst pneumonia is a major health problem worldwide, it is particularly so in developing countries. Treatments, prevention, and diagnosis have become a burden to poor governments in various continents. ${ }^{1}$ Whilst there are several aetiologies associated with pneumonia ${ }^{2}$ the most important single pathogen for this deadly disease is Streptococcus Pneumonia. ${ }^{3}$ These Gram-positive alpha haemolytic bacteria are commonly found in the nasopharynx of elderly people and young children. ${ }^{4}$ The irrational use of antibiotics in Somali regional state was researched using cross-sectional study. Two forms of questionnaires were developed Q1 and (Q2 in which will be explained later in the article). Using Q1 exactly 500 patients were interviewed of which 384 responses were valid and the rest were withdrawn for various reasons. Misuse of antibiotics for Pneumonia and other infectious diseases were presented and observed in this study which showed that more than $50 \%$ of respondents did not complete the antibiotics used for pneumococcus treatment such as Ceftriaxone or Augmentin for example. Confusion occurs as to the best form of treatment for pneumonia in the Somali state of Ethiopia since there are no functioning common methods and guidelines. Ideally these should be implemented by professionals then monitored and evaluated by Ethiopian authorities. The Ethiopian government has prepared excellent policies and guidance for managing treatment of disease in all Ethiopian hospitals. The great challenge, however, is to implement their written policy across the board.
\end{abstract}

Keywords: Streptococcus, pneumonia, malnutrition
Volume 7 Issue 2 - 2019

\author{
Abdirahaman Awale Hassan \\ Ph.D in Clinical Research, Texila American University, South \\ America
}

Correspondence: Abdirahaman Awale Hassan, Ph.D in Clinical Research, Texila American University, Guyana, South America, Email awale_hassan@hotmail.com

Received: February 12, 2019 | Published: March 19, 2019

\section{Introduction}

Medicinal usage is rational, and it is a necessity of life for all animals including humans. This, however, is true only when patients receive appropriate medicines in the right conditions and for the correct length of time. ${ }^{5}$ Patients should receive this correct dosage to establish their wellbeing. On the other hand, irrational or incorrect use of medicines develops when one or more of these conditions are not met. ${ }^{6}$ Due to a lack of regular and up-to-date awareness training, the irrational use of antimicrobial agents is prevalent in Jijiga, the Somali regional state capital city. For this reason, this study aimed to investigate, evaluate and eventually to design a tool to reduce the widespread misuse of prescribed medication to treat pneumonia and related infectious diseases in this area. Another aspect of irrational use is the inappropriate taking of these drugs without medical advice. Courses of antibiotics which have been obtained over the counter or from illegal importers without prescription may be taken unnecessarily without an official diagnosis of pneumonia, and this adding to the danger of widespread resistance to antibiotics by unnecessary overuse.

According to Desta ${ }^{7}$ it is estimated that more than $50 \%$ of the medicines in the world that are prescribed, dispensed or sold are used inappropriately. ${ }^{8}$ Furthermore, it has been estimated that at least half of all patients around the world fail to consume prescribed or dispensed drugs as instructed by their professionals. ${ }^{6}$ The irrational use of medications can take various forms that contribute to such a prevalence of the misuse of medicines. These practices can be for instance, polypharmacy, obtaining medication without professional guidance, the over-use/underuse of antibiotics and usage of over the counter (OTC) medications. ${ }^{8}$ Failure of patients in Somali region to follow the advice of the health professionals, carelessness from health professionals in giving advice when issuing medication to the patient, not prescribing in accordance with clinical guidelines and inappropriate self-medication by patients will all contribute to compromising individual health outcomes and will enhance an already widespread antibiotic resistance ${ }^{8}$ Cost to the individual and to the community is another issue to be simultaneously addressed during this research. Sources need to be identified and regulated and prescriptions made from reliable diagnoses to reduce the current burden of medication costs to government and community resources.

\section{Conceptual definitions}

Antibiotic misuse or abuse: refers to the overuse of antibiotics with potentially serious effects on health.

Irrational use of medicine: is a global phenomenon that is the opposite of the rational use of medicine where patients receive medications that are appropriate to their clinical needs in the right dose for the correct duration at an appropriate cost.

Pneumonia incidence and prevalence: There are about 3 million reported cases of pneumonia per year in the United States with about 60,000 fatalities. About one-third of pneumonia cases occur in people over age 65 .

\section{The relationship between malnutrition and pneumonia in children}

Malnutrition is considered to be one of the most serious health problems in developing countries. It commonly affects infants and young children under 5 years of age and is the most important 
underlying risk factor that is responsible for the death of many children in the African continent. Under nutrition specifically increases the frequency and severity of pneumonia episodes, finally leading to the presentation of secondary immune deficiency. ${ }^{9}$ The underlying mechanisms are still inadequately understood, as well as why different types of malnutrition are associated with different diseases. ${ }^{10}$ For example, malnutrition can be considered to be at the base of several clinical conditions including kwashiorkor, marasmus, wasting, stunting, and micronutrient deficiencies. Whilst various studies suggest that particular nutritional deficiencies are strongly associated with approximately half of all deaths in children, ${ }^{11}$ as can be seen here, there is no doubt as to the significant and direct impact of malnutrition pneumonia on mortality. Approximately, 77 million children are born every year in Africa Asia and Latin America, and about 4.7 million children of the 77 million die every year of pneumonia malnutrition related causes.

\section{Anti-bacterial resistance}

The bacteria are clever enough to protect themselves against the drug, using various tactics to prevent antibiotics from attacking them. The benefit of antibiotics is uncountable and thus antibiotics have become in recent years one of the most important medical interventions needed for the development of complex medical approaches such as cutting-edge surgical procedures, solid organ transplantation, and management of patients with cancer, among others. Nonetheless, an increase in antimicrobial resistance amongst most bacterial pathogens is hindering and threatening this therapeutic achievement, endangering the successful outcomes of critically ill patients. In fact, the World Health Organization has named antibiotic resistance as one of the three most important public health threats of the $21^{\text {st }}$ century. This bacterial resistance has created an economic burden around the world, USA alone spending 120 million per year to overcome this problems. In Africa economic hardship is growing and fighting against bacterial resistance is affecting the socio-economics of east Africa. Prevalence of bacterial resistance can be attributed to various reasons such as over use and direct misuse of antibacterial products or antibiotics

\section{Literature review}

The abuse of antibiotics internationally versus in Somali regional state, Ethiopia

Despite the inappropriate use of antibiotics being a global problem there are some countries which are largely incapable of monitoring medicinal usage or sufficiently taking action to correct the misuse of the medications. ${ }^{6}$ Most European and North American countries are doing well in controlling medicinal misuse, as they have succeeded in implementing the regulations and guidance relating to medication. ${ }^{6}$ The correct usage of a medicinal product is essential to alleviate symptoms and to promote wellbeing whereas misuse of drugs can be life threating and increase the level of mortality. Several studies have suggested that irrational use of medicines has been dramatically increasing over recent decades, particularly in the developing countries of Africa and Asia. This particular study has been subject to various limitations. Essentially and firstly, since there are no similar studies done in Somali regional state, Ethiopia, then this study will be unique of its kind and therefore there is no literature available in search engines and databases.

Secondly, due to security issues and political unrest that recently erupted in the region, we were not able to include many places and cities within the Somali regional State in our research. Thirdly, no financial assistance was obtained from anyone and thus our movement was limited to Jigjiga and surroundings for lack of funding.

Fourthly, apart from the permission to collect data we did not receive any support from government agencies and others, and consequently this study was limited to a convenience sampling method to select the respondents in Jijiga, the capital city of Somali regional State. Data presented in this study therefore is not suitable for application to the overall population in Ethiopia or Somali Regional State (SRS).

\section{Rationale \& need for the study}

In the Somali region there has been a marked tendency to overuse medication but until now no independent study had been carried out in the region to evaluate the level of prevalence of antibiotic misuse. After completion of this research awareness and training must be followed.

\section{The research question}

To assess the level of antibiotic misuse in the region and the need to create a tool or method to tackle these unwanted behaviours in order to maximise the effect of antibiotics and to reduce the cost of medicine in the region.

\section{Objectives}

The aim of this study was to investigate the level of prevalence of antibiotic misuse in general and specifically the antibiotic such as ceftriaxone, Augmentin, doxycycline and procaine penicillin used against pneumonia in Somali regional State. To evaluate how the rules and regulations for medicinal use and public safety are implemented and observed in Somali regional State and to provide solutions for the misuse of antibiotic use in this area.

\section{Hypothesis}

Irrational use of medicine in general is widespread in Jijiga, the capital city of Somali regional State of Ethiopia as well as other places in the region, and the uninformed usage of antibiotics for suspected pneumonia and related diseases is very high due to ignorance of the patients and the lack of implementation for the written guidelines from the Ethiopian government. Once obtained from whatever source, and assuming that a related disease is present, the drugs are then not necessarily used correctly: in the right amounts, sufficiently regularly or for the correct period of time. Any or all of these inaccuracies may be present.

\section{Methodology}

A cross-sectional study was conducted to evaluate awareness, attitude and practices of the private drug shops and pharmacies in the Somali Regional State capital city (Jigjia) Ethiopia. Our study evaluates the general skills of professionals and public knowledge of the use of antibiotics in general and antibiotics used to treat pneumonia in particular such as Ceftriaxone and Augmentin. Specifically, the contribution of drug misuse towards antibacterial resistance is considered. Specifically, this study assesses the behaviour of private pharmacies, drug shop owners and governmental pharmacy attendants concerning drug handling and management issues and to identify the condition of the drug supply chain in the region. This study was conducted during the period between $28^{\text {th }}$ May 2018 and $2^{\text {nd }}$ August 2018 in the Somali region of Ethiopia. The Somali regional 
state consist of nine zones, the capital city of the region is called Jijiga which is about $716 \mathrm{KM}$ east of Addis Ababa, the capital city of Ethiopia. We have chosen Jijiga due to the availability of resources and there being enough pharmacies and drug shops in the capital for analysis.

The population of this study was initially intended to consist of 500 individuals from the public who were either current patients or previous patients of one of the clinics, hospitals, and pharmacies in the capital city of Somali regional state in Ethiopia plus 36 professionals from various businesses within the pharmaceutical sector. Unfortunately, only 384 interview papers became valid and the remaining 116 were excluded from these studies for a variety of reasons, including personal decisions, age restriction, employment and financial issues. Therefore 384 individuals finally participated in this study. Prior to beginning the study, literature reviews have been extensively done on the topic. Finally the decision was made to develop and design three questionnaires. ${ }^{12-14}$ Section one concerned the respondent's personal information including gender, age, marital status, ethnicity, education level and employment status.

\section{Measures/tools used for data collection}

A separate and structured questionnaire (Q2) was prepared and administered to governmental pharmacies, the private drug shop owners and public pharmacy attendants, to assess the knowledge, attitude and practice of the professionals towards drug handling, management and supply chain issues. The questionnaire was pretested in the field to check for clarity and cultural acceptance of the ideas and relevant issues were incorporated in an open-ended form. Verbal consent was obtained from the respondents after the objectives of the study were explained to them before starting the interview. Data collected from all the surveyed area using the standard data collection forms were coded, checked for accuracy, consistency, omissions, and irregularities. They were then entered into Microsoft Excel 2010 and summarised using the standard summary forms.

\section{Ethical considerations}

Prior to the initiation of this project, a letter was sent to the Drug Administration and Control Authority requesting their permission for the investigation and research to take place in the aforementioned location. The regional government and other concerned agencies eventually permitted us to begin our research without restrictions. DACA is the only government office that is responsible for drug control and the drug regulatory office which is a branch of FMHA DACA that deals all drugs consumed in this region. The branch is located in the capital city of Somali regional state known as in Jigjiga.
Brief explanations were also given on the objectives as well as the benefit of the study to the concerned officials and their verbal consent was obtained. Each respondent who was interviewed was asked to give their consent after the purpose, objective, and benefit of the study had been explained to them. The confidentiality and privacy of every respondent's information was ensured, all participants were volunteers and no financial interests were intercepted during the study.

\section{Results}

This study showed that almost all of the participants $89 \%$ have not completed their medication, $193(50.2 \%)$ respondents stopped medication before completing treatment because they feel that their health improved and symptoms alleviated. Equally, about 61 people out of 384 patients, (16. \%) did not complete the antibiotics for pneumococcus treatment for various reasons and only $42(11 \%)$, of respondents, have successfully completed their medication and had no left-over drugs at home. Some respondents, $(8 \%)$, said due to forgetfulness they had left over drugs present at home. A similar finding was in fact reported in India, which showed $165(70 \%)$ participants had up to five expired medications at home and the major reason for possession of unused, expired Ceftriaxone or Augmentin was that they were purchased over the counter (OTC) drugs for selfmedication with related excess supply of medications. In general, about $89 \%$ of the interviewed households were not able to finish medication for various reasons (Table $1 \&$ Table 2).

Table I Reasons why people do not complete the course of medication, Total respondents $\mathrm{N}=384$

\begin{tabular}{lll}
\hline Category & Frequency & (\%) \\
\hline Subsiding of the condition & 193 & $(50.2 \%)$ \\
Combined reasons & 61 & $(16.0 \%)$ \\
No left-over drugs & 42 & $(11 \%)$ \\
Forgetfulness & 8 & $(2.1 \%)$ \\
Excess supply & 15 & $(4 \%)$ \\
Intolerable side effects & 15 & $(3.8 \%)$ \\
Passed expiry date & 11 & $(2.8 \%)$ \\
Changed treatment & 8 & $(2 \%)$ \\
Unclear instruction & 4 & $(1 \%)$ \\
Unpleasant taste & 8 & $(2 \%)$ \\
Others & 15 & $(3.8 \%)$ \\
\hline
\end{tabular}

Table 2 Socio-demographic data of the respondents calculated in single factor

\begin{tabular}{lllll}
\hline ANOVA: Single Factor & & & & \\
\hline Summary & & & & \\
\hline Groups & Count & Sum & Average & Variance \\
\hline Age & 6 & 384 & 64 & 1778.8 \\
Gender & 6 & 384 & 64 & 9850 \\
Ethnicity & 6 & 384 & 64 & 12878.8 \\
Education & 6 & 384 & 64 & 3390 \\
Occupation & 6 & 384 & 64 & 3813.2 \\
Marital Status & 6 & 384 & 64 & 6490.8 \\
Diagnosed with pneumonia & 6 & 384 & 64 & 13594 \\
\hline
\end{tabular}


Table Continued....

\begin{tabular}{lllllll}
\hline ANOVA & & & & & & \\
\hline Source of Variation & SS & df & MS & F & P-value & F crit \\
\hline Between Groups & 0 & 6 & 0 & 0 & 1 & 2.371781 \\
Within Groups & 258978 & 35 & 7399.371 & & & \\
Total & 258978 & 41 & & & & \\
\hline
\end{tabular}

Figure 1 compares the difference between various pharmacists towards their behavior in inspecting their stock if they do selfinspection rather than waiting any governmental agency to inspect them.

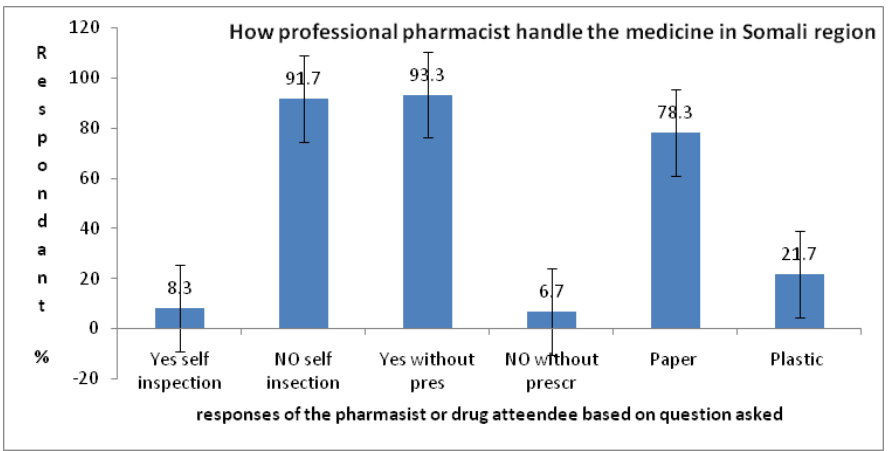

Figure I How professional pharmacist handle the medicine in Somali regional State.

\section{Pharmacists' administration and advice concerning} drugs:

The above graph (Figure 2) explains the behaviour of the pharmacist in the service with regard to the handling and storage of Cephalosporins and Ceftriaxone, drug disposal after the expiration date, the sort of advice that patients receive during obtaining medicine from the pharmacist and if there are any follow ups from either doctors or pharmacists. The Graph shows that every patient has been advised in some degree, as each pharmacist instructed the patients how to use the drugs, i.e they have clearly indicated if the drug is used as PO, IM, IV or other means of drug administration, such as taking once a day (24hrs), BID or TID etc. However, they did not explain any side effects that can occur during the course and no further advice was given to the patients. In addition, the scale of follow up by the professionals appears to be inadequate as $(73.3 \%)$ of the population were not contacted after dispensing. Only $(26.7 \%)$ were contacted for follow up. This indicates a reduced level of awareness in comparison with other countries. It would seem clear that a very small number of patients were communicated by either pharmacist or physicians after dispensing.

Effective drug handling and drug management are crucial for the safety of both patient and public. It can be seen from this chart (Figure 3) that (76.7\%) of pharmacists in our sample and those who sell medication in the region have reported that they do not store the medicine according to manufacturer's recommendations. Only $(23.3 \%)$ have followed the guidelines of manufacturers. As is clear from this data, the majority of pharmacists in the region are using their own method for storing, lacking proper guidance from the Ethiopian authority. This could lead to drug-resistance and the poisoning of vulnerable people and animals in the region if no immediate intervention is taken.

The above chart (Figure 4) shows the sources of medicine used in Somali regional state of Ethiopia. This information was received from DACA regional office, based on research carried out by DACA and they have published their handbook which is archived in their main office in Jijiga, the headquarters of The Somali regional state of Ethiopia. Half of the pharmaceutical products utilized in the region were received either directly from the federal government or other affiliated sources of government. All drugs from central government are manufactured in Ethiopia as the government controls local product and drug control management. This shows the majority of drugs are controlled by the central government (Figure 5).

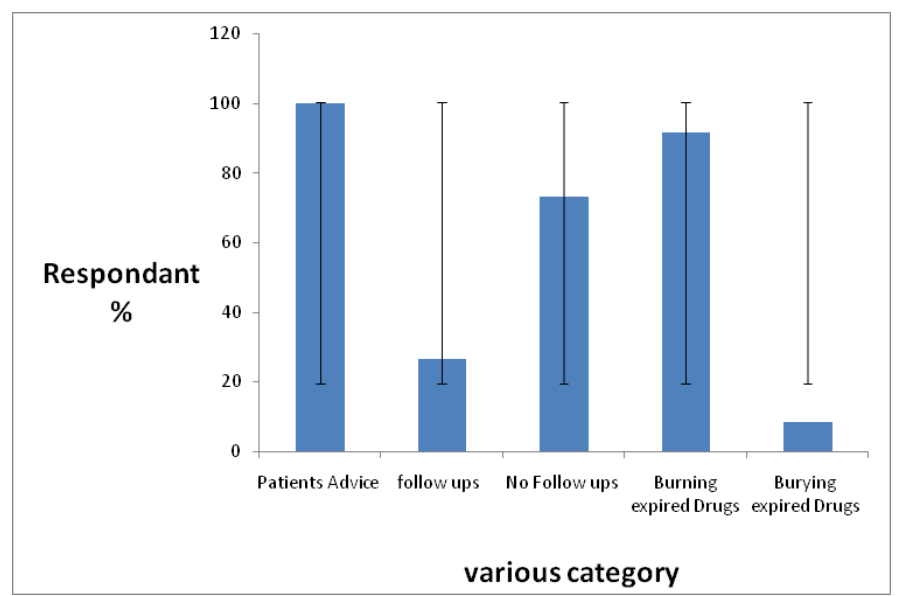

Figure 2 Drug disposal patient advice and follow up.

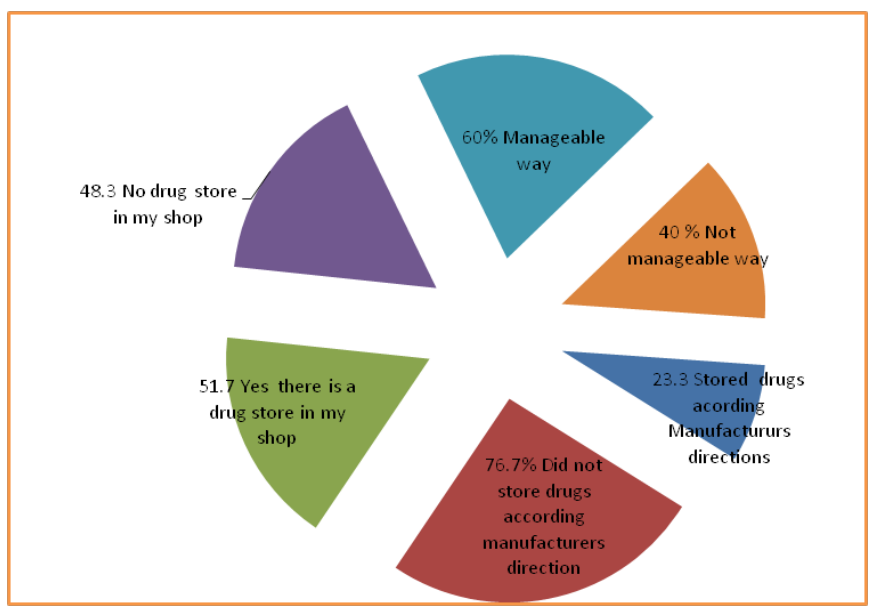

Figure 3 Drug handling and store management.

Nonetheless, $15 \%$ are from Non-governmental organizations including both local NGO like OWDA (Ogden Welfare and Development Association), and other international organizations such as UNHCR, WHO NRC Save the Children and many other organizations who are present in the region. About $9 \%$ of the medications in the region were imported from other countries mainly from Korea, Europe, India, China and Arab countries. 


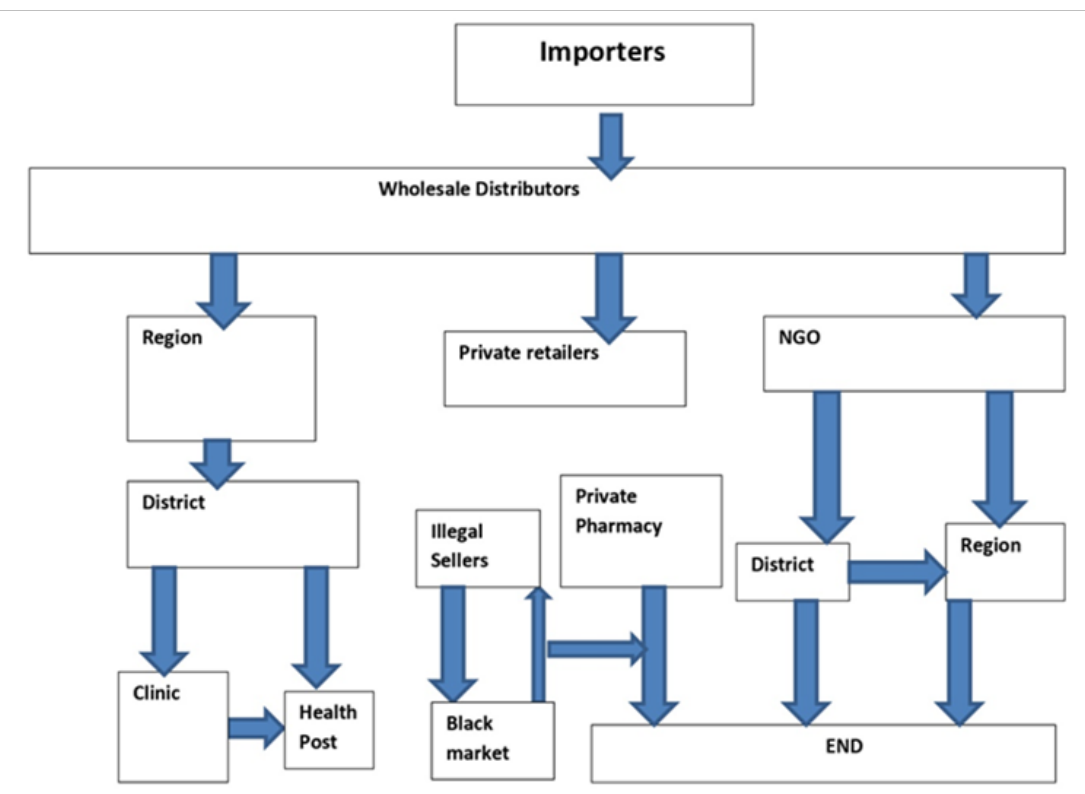

Figure 4 Resources of medicine utilized in Somali Regional State, East Ethiopia.

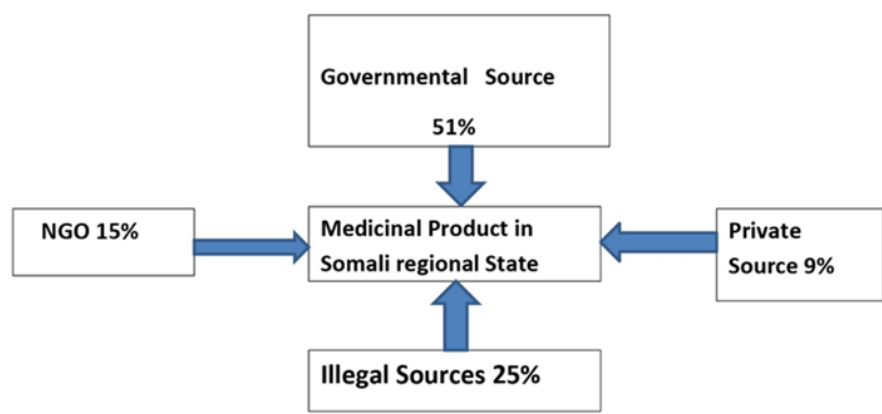

Figure $\mathbf{5}$ The illegal traffic in medicinal drugs and associated financial problems.

Finally, 25\% of the total of medicinal products consumed in the region were found to be illegally procured and the drug control office seems to be aware that a considerable amount of drugs are being smuggled daily from other countries including Europe through borders attached to Ethiopia. This illegal medication mainly passes the border via Somaliland with its "self-declared government". There are many factors that contribute to the irrational of medicinal product in the Somali region of Ethiopia; this could be lack of updated workshops, training and awareness activities which highlight proper usage of Ethiopian guidelines. Medicine coming into the region is in large part from illegal sources. There are considerable amounts of medicinal products that get into the region illegally.

Non-governmental organizations (NGOs) and Ethiopian governments also are the part of the problem rather being part of the solution as they employ inadequately experienced people to handle medicine being mainly other Ethiopian nationals who were not trained enough in the area. Drug disposal, drug handling, transportation process and giving advice to the end users are all being performed ineffectually, exacerbating the situation and thus hugely contributing to irrational drug usage and antibacterial resistance in tackling pneumonia in the region. However whilst this is an important problem to address, a significant aim of this study was to determine the level of public awareness in utilising drugs and medication. Various strategies were employed to find out where the real problems lie.

\section{Lack of compliance with instructions for use}

\section{People buy antibiotics on their own advice}

It is widespread to see people using medication for any illness in general and usage of antibiotics for pneumonia and other infections without seeing a doctor or undergoing laboratory investigations. In this study, we determined to know the level of prevalence for using the drug without a prescription. According this study, 54\% of the patients did not complete their medication for a variety of reasons (Table 1). $75.3 \%$ of those interviewed who used antibacterial agents stated that they were not diagnosed with pneumonia despite using antibiotics from drug shops mainly Ceftriaxone (g, IV), or Augmentin $625 \mathrm{mg}$ without thinking about the consequences of using antibacterial agents as Table 2 indicates p. Value of this data is greater than 0.05 and therefore this suggesting the null hypothesis must be rejected as this is statistically not significant as result shown in Figure 2. (Anova: Single Factor)

\section{People stop taking the antibiotic before the course is finished}

Either because they a) lacked any advice by buying the drugs without prescription, or were not given advice by medical personnel on being prescribed the drugs, or they did not understand the importance of the advice and ignored it, stopping the drug when they felt an improvement. Usage of pharmaceutically active agents requires professional advice from healthcare professionals and overdose can cause serious harm to human lives, similarly, inadequate use of medicine will definitely influence its effect and this certainly will contribute antibacterial resistance.

\section{Lack of adherence to instructions on dosages}

This study clearly showed that the respondents stated low obedience in terms of stopping their prescribed medicines as soon as they felt recovered or symptoms alleviated even temporally. This study completely agrees with what was found in other studies done in Oman that concluded that people do not adhere to their treatment regimens. ${ }^{15}$ 


\section{Lack of clear instructions from medical personnel}

One of the most imperative factors for this study was the use of medication without proper advice and also a lack of prescriptions. This study involved the extent to which professionals neglect the Ethiopian guidelines for the safety and wellbeing of patients in the region. During the study, it has been identified that professional doctors, nurses, pharmacist are all lacking awareness of and mainly contribute to the irrational use of medicines. This potentially also has a negative impact on antibiotic resistance in the region.

\section{Reconsideration of the length of dosage is needed}

Having criticised the early termination of a course of treatment by patients, it may be useful to consider at this point that there is a case for administering short dosages which are possibly more desirable than prolonged ones for several reasons, including price, time consideration and the minimising of side effects. In addition, a longer period of treatment may tend to result in unfinished medication resulting in many medicines being leftover.

On the other hand, overly quick withdrawals of medicine can attract antibacterial resistance and any bactericidal effect will be hindered and as a consequence undesirable bacteria might survive. According to the Centre for Disease Control and Preventions (USA), stopping antibiotics as soon as the patients feel better can result in the need to resume treatment later and as a result this might lead to antibiotic resistance as full treatment is necessary to kill bacteria (bactericidal) or inhibit (bacteriostatic) the growth of bacteria.

To stop or quickly withdraw from prescribed medication has become popular in the region as this study indicates about $50.2 \%$ of the participants responded that they have completely stopped their medication due to improvements of their condition and this study is in line with other researches done by Centre of Disease Control and prevention in America, 193 out of 384 is a considerable population which clearly sends a message to Ethiopian authority and International organisations who are present in the region to investigate this matter and create tools to educate people and monitor the development of this system. As mentioned this kind of behaviour is very common in other parts of the world ${ }^{1}$ including European countries, study done Romania concluded similar findings with our results similarly in Malaysia people have majority of tendency to stop medication because they got better and pain has gradually dulled. ${ }^{4}$ However, the studies mentioned here are not specifically referring to antimicrobial agents but to medicine generally including antipyretics and vitamins. ${ }^{15}$

\section{Appropriate storage and allocation}

The vast majority of the professionals declare that they usually sell and dispense antibiotic medicine to patients without prescription from either hospitals or clinics. Exactly (93.3\%) have stated so, in comparison with other study done in Spain which shows that dispensing drugs without the necessary prescription is at about $(10.8 \%)^{16}$ in which they consider is very high and is not restricted to any particular drug therapy group. Doctors, dentists, nurses, pharmacists, patients and health service management must all assume their share of responsibility. ${ }^{16} 93 \%$ demonstrates a very large number of the pharmacists or drug attendees in Jigjiga to have replied and stated that they dispense medicine without prescriptions. Surprisingly, in another study done in elsewhere only $(11.1 \%)$ of prescription drugs were requested without a prescription ${ }^{16}$ in this study only a small proportion have said they need a prescription from a physician $(6.7 \%)$ which is very small to compare to $(93.3 \%)$ of pharmacists who produce medication to patients or clients without asking if they have prescription. Figure 1 Shows that $(78.3 \%)$ of the respondents have said they have given dispensed the medication with intact papers and boxes while (22.7\%) of them have used plastics for handling medicine.

\section{Correct storage and disposal of drugs}

Regarding the proper storing of medicine which is essential for the health and safety of the community, the use of medicine until the expiry date elapses is rational. Nonetheless, due to the lack of information on some of the medication used in Ethiopia resulting from labelling problems, it is very difficult for many people to know what date is the expiry date for medications. Concerning physical storage, Figure 2 Shows that (78.3\%) of the respondents has said they have dispensed medicine to their patients with intact papers and boxes while $(22.7 \%)$ of them have used plastics for handling medicine. As to the disposal and handling of expired drugs, Figure 5 shows that the majority of interviewed individuals have burned the expired medication although it is the responsibility of the government to arrange the disposal facility, This study also supports and is clearly in line with a cross-sectional study done in Kabul Afghanistan in which almost the entire sample $(98 \%)^{2}$ of the participants felt that improper disposal of unused and expired medicines can affect the environment and public health.

\section{Conclusion}

The Ethiopian guidelines for drug prescription and drug dispensing that the DACA requests to be used as a national guideline for all the regions in this country is adequate enough to improve the quality of life of patients in the all regions. ${ }^{17}$ However, the attitude, the knowledge and the awareness of both health professionals and patients are more imperative than well-scripted guidelines that are not in use. A new approach to tackle this problem is mandatory to save many lives wasted due to preventable behaviour. It is important to design a new approach that can improve the quality control for medicines utilised in the region and which will enhance the quality of antibiotic usage within the community. ${ }^{18}$ Regular training about the negative effect of unfinished medication and its exposure to the environment is important. In an attempt to reduce the prevalence of unfinished medication we aim to request the pharmacist or drug shop attendee to give patients who complete their medication a reward such as an activity, discount or draw prize and anyone who destroys medication in the environment will face negative consequence such as a small fine. This, however, can only happen when federal government, local government, NGO's and environmental agencies agree to proceed and participate in the intervention. This study can be the base for any new research as is no single article written about antibiotics, medicine disposal, drug control or similar subjects in the entire region. ${ }^{19-22}$ It is very unfortunate to note that this region was marginalised in many aspects, particularly where health issues are concerned. I have searched all search engines and found no articles about these topics written about this region, I am so glad to carry out this study expecting that it will be an essential building block for further studies and I urge the scientific community to extend their research and to include the neglected regions in the world such as Somali Region of Ethiopia.

\section{Acknowledgments}

None.

\section{Conflicts of interest}

Author declares that there is no conflict of interest. 


\section{References}

1. Bhutta ZA. Childhood peumonia in developing countries, Rerinement of clinical algorthms is a priority. BMJ. 2006;333(7569):612-613.

2. Hassan Abdirahman. Is Lactate an undiscovered pneumococcal virulence factor? Texila Journal. 2015;2(2):1-5.

3. Yesilkaya H, Spissu F, Carvalho SM, et al. Pyruvate formate lyase is required for pneumococcal fermentative metabolism and virunlence. Infect Immun. 2009;77(12):5418-5427.

4. Kadioglu A, Weiser JN, Paton JC, et al. The Role of streptococcus pnemoniae Virulence factors in host respirotory colonizationand Disease. Nat Rev Microbiol . 2008;6(4):288-301.

5. Revention and Containment of Antimicrobial resistance. WHO; 2012. 56

6. Gualano MR, Gili R, Scaioli G, et al. General population's knowledge and attitudes about antibiotics: a systematic review and meta-analysis. Pharmacoepidemiol Drug Saf. 2015;24(1):2-10.

7. Desta AH. Veterinary Drugs Handling, Management and Supply Chain Assessment in Afar Pastoral Region of North East Ethiopia. American Journal of Bioscience and Bioengineering. 2015;3(6):142-148.

8. The World Medicine Situation. Third Edition. Geneva: WHO; 2011.

9. Wiens MO, Pawluk S, Kissoon N, et al. Pediatric post-discharge mortality in resource poor countries: a systematic review. PLoS One. 2013;8(6):e66698.

10. Rytter MJ Kolte L, Briend A, Friis H, et al. The immune system in children with malnutrition-a systematic review. PLoS One. 2014;9(8):e105017.

11. Committing to Child Survival: A Promise Renewed. UNICEF; 2015. 104
12. Braund R, Peake BM, Shieffelbien L. Disposal practices for unused medications in New Zealand. Environ Int. 2009;35(6):952-955.

13. Vollmer G. Disposal of pharmaceutical waste in households-a European Survey. Green and Sustainable Pharmacy; 2010. 165-178 p.

14. Wu M, Atchley D, Greer L, et al. Dosed Without Prescription:Preventing Pharmaceutical Contamination of Our Nation's Water. Natural Resources Defense Council; 2009. 4 p.

15. Abdo-Rabbo A, Al-Ansari M, Gunn BC, et al. The use of medicines in Oman: public knowledge, attitudes and practices. Sultan Qaboos Univ Med J. 2009;9(2):124-131.

16. Barbero-González A1, Pastor-Sánchez R, del Arco-Ortiz de Zárate J, et al. Demand for dispensing of medicines without medical prescription. Aten Primaria. 2006;37(2):78-87.

17. Standard treatment guidelines for zonal hospitals. FMHACA; 2014.

18. Teni FS, Birru EM, Surur AS, et al. Pattern and predictors of medicine use among households in Gondar Town, northwestern Ethiopia: a communitybased medicine utilization study. BMC Res Notes. 2017;10(1):357.

19. Bashaar M, Thawani V, Hassali MA, et al. Disposal practices of unused and expired pharmaceuticals among general public in Kabul. $B M C$ Public Health. 2017;17(1):45.

20. Dawood OT, Hassali MA, Saleem F. Factors affecting knowledge and practice of medicine use among the general public in the State of Penang, Malaysia. J Pharm Health Services Res. 2017;8(1):51-57.

21. Justina G, Guy W Soo Hoo. Bacterial Pneumonia Medication. Medscape; 2017.

22. The Pursuit of Responsible Use of Medicines: Sharing and Learning from Country Experiences. Geneva: WHO; 2012. 78 p. 